Cochrane Database of Systematic Reviews

\title{
Mindfulness for smoking cessation (Protocol)
}

Jackson S, Brown J, West R, Norris E, Livingstone-Banks J, Lindson N

Jackson S, Brown J, West R, Norris E, Livingstone-Banks J, Lindson N.

Mindfulness for smoking cessation (Protocol).

Cochrane Database of Systematic Reviews 2020, Issue 7. Art. No.: CD013696.

DOI: 10.1002/14651858.CD013696.

www.cochranelibrary.com 
TABLE OF CONTENTS

HEADER 1

ABSTRACT

BACKGROUND

OBJECTIVES

METHODS

ACKNOWLEDGEMENTS

REFERENCES

APPENDICES

HISTORY

CONTRIBUTIONS OF AUTHORS

DECLARATIONS OF INTEREST

SOURCES OF SUPPORT 
[Intervention Protocol]

\section{Mindfulness for smoking cessation}

Sarah Jackson ${ }^{1}$, Jamie Brown ${ }^{1}$, Robert West ${ }^{1}$, Emma Norris$^{2}$, Jonathan Livingstone-Banks ${ }^{3}$, Nicola Lindson ${ }^{3}$

1Department of Behavioural Science and Health, University College London, London, UK. ${ }^{2}$ Centre for Behaviour Change, University College London, London, UK. ${ }^{3}$ Nuffield Department of Primary Care Health Sciences, University of Oxford, Oxford, UK

Contact address: Sarah Jackson, s.e.jackson@ucl.ac.uk.

Editorial group: Cochrane Tobacco Addiction Group.

Publication status and date: New, published in Issue 7, 2020.

Citation: Jackson S, Brown J, West R, Norris E, Livingstone-Banks J, Lindson N. Mindfulness for smoking cessation (Protocol). Cochrane Database of Systematic Reviews 2020, Issue 7. Art. No.: CD013696. DOI: 10.1002/14651858.CD013696.

Copyright @ 2020 The Cochrane Collaboration. Published by John Wiley \& Sons, Ltd.

\section{A B S T R A C T}

\section{Objectives}

This is a protocol for a Cochrane Review (intervention). The objectives are as follows:

To assess the efficacy of mindfulness interventions for smoking cessation among people who smoke, and whether mindfulness interventions for smoking cessation have an effect on mental health outcomes. 


\section{B A C K G R O U N D}

\section{Description of the condition}

Smoking remains a leading cause of preventable death and disease worldwide, causing over eight million deaths each year (WHO 2019). Stopping smoking can result in substantial health gains, even later in life. The sooner a smoker quits, the more they reduce their risk of developing smoking-related diseases (Doll 2004). The majority of smokers want to quit and many try to quit each year, but quit rates remain low (WHO 2019).

\section{Description of the intervention}

In recent decades, mindfulness has increasingly been recognised as an influence on mood and behaviour (Baer 2003; Keng 2011). It has been adopted as an approach for increasing awareness and responding skilfully to mental processes that contribute to emotional distress and maladaptive behaviour (Baer 2003). In current research contexts, mindfulness is typically defined as the psychological process of bringing non-judgmental attention to experiences occurring in the present moment (Kabat-Zinn 2013). A two-component model has been proposed, operationalising mindfulness as: (i) maintaining attention on the immediate experience, and (ii) maintaining an attitude of openness, curiosity, and acceptance toward this experience, regardless of its valence or desirability (Bishop 2004).

Mindfulness approaches are not relaxation or mood management techniques, but rather a form of cognitive training to reduce susceptibility to reactive states of mind that might otherwise induce stress or perpetuate psychopathology (Baer 2003). According to these approaches, the ability to practice mindfulness is developed using various meditation techniques that originate from Buddhist spiritual practices (Nhất Hanh 1987). The practice of mindfulness meditation involves focusing attention on the experience of cognitions, emotions, perceptions, and sensations, and simply observing them as they arise and pass away. A key tenet of mindfulness is that, by simply noticing thoughts and feelings in a curious and accepting manner, people develop greater tolerance of these phenomena and are able to recognise that they are transient, so they are less likely to respond impulsively to them (Heppner 2015).

The two foremost treatments that are explicitly based on mindfulness are Mindfulness-Based Stress Reduction (MBSR; Kabat-Zinn 2013) and Mindfulness-Based Cognitive Therapy (MBCT; Segal 2002). MBSR was developed to treat chronic stress and pain-related disorders, and $\mathrm{MBCT}$ was developed to prevent relapse in depressive disorders. Both of these approaches use meditation as the primary method of teaching mindfulness. Other treatments that incorporate mindfulness include Acceptance and Commitment Therapy (ACT; Hayes 2016), Dialectical Behaviour Therapy (DBT; Linehan 2018) and Marlatt's Relapse Prevention model (Marlatt 2005).

\section{How the intervention might work}

Mindfulness training may aid smoking cessation by teaching individuals to pay attention to, and work mindfully with, negative affective states, cravings, and other symptoms of nicotine withdrawal as they arise, rather than habitually reacting to these unpleasant states by smoking. Proposed mechanisms of action include attention regulation, body awareness, emotion regulation, and change in self-perspective (Hölzel 2011).

Withdrawal following smoking cessation is acutely associated with heightened levels of stress and negative affect (Shiffman 2004; West 2017). Once withdrawal symptoms have abated, cessation is generally associated with improved mental health (Taylor 2014), but early stage acute stress, negative affect, and depression are predictive of relapse (Correa-Fernández 2012; Glassman 1990; Shiffman 2004; Shiffman 2005). Therefore, interventions that work to reduce these adverse emotional consequences of stopping smoking may enhance quit rates and ultimately prevent relapse. Mindfulness interventions have shown some efficacy in the treatment of psychiatric disorders relating to or involving these negative affective states (Goyal 2014; Marchand 2013).

Further, by teaching smokers to focus their attention on what is happening in the moment, mindfulness training brings habitual behaviours into consciousness. This enables people to understand the associative learning process, and focus on affect and craving as central components of positive and negative reinforcement loops (Brewer 2010). By emphasising the transience of affective states and teaching smokers to 'sit with' negative affect and craving, mindfulness interventions target and modify learned responses to smoking cues. This may help smokers to quit, and may reduce cigarette consumption among those who do not stop smoking completely.

Thus, it has been suggested that mindfulness-based treatments "may have the relative advantage of teaching a single technique that may lead to the dampening and eventual dismantling of the complex interrelated associative processes of smoking rather than just removing stimuli that might propagate them" (Brewer 2011).

\section{Why it is important to do this review}

If found to be effective, mindfulness-based treatments could add an innovative and important intervention option to the range of treatments for smoking cessation. A systematic review, including literature to 2016 , did not find evidence of a significant impact of mindfulness meditation interventions on abstinence relative to comparator groups (Maglione 2017). However, the evidence identified was of low certainty due to the high levels of heterogeneity and imprecision detected through meta-analysis. Therefore, there is a need to update this review to include new evidence, in an effort to increase the certainty of the resulting conclusions. The purpose of the present review is to assess the effect of interventions that incorporate mindfulness approaches for smoking cessation, using the robust methodology of Cochrane and the Tobacco Addiction Group. This review also represents part of a separate project to evaluate similarities and differences between the standard methodological processes of the Cochrane Tobacco Addiction Group and a novel, machine-learning approach developed by the Human Behaviour Change Project (HBCP; Michie 2013).

\section{O B JECT IVES}

To assess the efficacy of mindfulness interventions for smoking cessation among people who smoke, and whether mindfulness interventions for smoking cessation have an effect on mental health outcomes. 


\section{METHODS}

\section{Criteria for considering studies for this review}

\section{Types of studies}

Randomised controlled trials (RCTs) and cluster-RCTs that measure smoking cessation at least six months from baseline are eligible for this review. We will include studies reported as full text, those published as abstract only, and unpublished data, where available. There will be no language or date restrictions.

\section{Types of participants}

We will include current tobacco smokers of any age who are willing to enrol in a smoking cessation trial. We will exclude trials that only recruit pregnant women, as their particular needs and circumstances warrant their treatment as a separate population, and these studies are covered in a separate Cochrane Review (Chamberlain 2017).

\section{Types of interventions}

We will include interventions targeted at tobacco smoking cessation that are either labelled as mindfulness, or involve a mindfulness component that can be isolated for analyses to investigate effectiveness. There will be no restrictions on the minimum duration of the intervention. Where a potentially relevant study intervention is not specifically described as being mindfulness-based, we will discuss as a team whether it is eligible for inclusion.

Eligible studies will include at least one of the following comparison (control) interventions:

- no smoking cessation treatment;

- another smoking cessation intervention, of any length or intensity (including usual care);

- another type of mindfulness intervention (e.g. mindfulness of a lower intensity).

\section{Types of outcome measures}

Our first primary outcome will be smoking abstinence at longest follow-up (dichotomous). To be eligible for inclusion, studies must measure abstinence at least six months from the start of the intervention. Following standard methods of the Cochrane Tobacco Addiction Group, we will exclude studies that only measure abstinence at less than six-month's follow-up.

In trials with more than one measure of abstinence, we will prefer the measure with the strictest criteria, in line with the Russell Standard (West 2005). We will use prolonged or continuous abstinence over point prevalence abstinence, and prefer biochemically-validated abstinence (e.g. using exhaled carbon monoxide or cotinine measures) over self-report. We will favour biochemically-validated point prevalence abstinence over self-reported continuous or prolonged abstinence.

Our second primary outcome will be mental health and wellbeing. This could provide us with information on potential benefits or harms of the mindfulness interventions. Even if comparisons of mindfulness interventions with other smoking cessation interventions do not find a benefit of mindfulness for smoking cessation, improved mental well-being could be a reason for choosing this treatment over another. We will assess validated measures of the following relevant constructs:

- depression;

- anxiety;

- quality of life;

- stress.

We will extract data on these mental health and well-being outcomes, measured at the longest follow-up at which abstinence is reported, or as close to this as possible. If meta-analysis is appropriate for this outcome, we will analyse these constructs separately.

\section{Search methods for identification of studies \\ Electronic searches}

We will search the Cochrane Tobacco Addiction Review Group's specialised register, Cochrane Central Register of Controlled Trials (CENTRAL), MEDLINE, Embase, and PsycINFO from inceoption, for trials that refer to mindfulness techniques in the title or abstract, or as keywords. The full MEDLINE search strategy is shown in Appendix 1. The Cochrane Tobacco Addiction Group's register has been developed from electronic searching of MEDLINE, Embase, PsycINFO and Web of Science, together with handsearching of specialist journals, conference proceedings and reference lists of previous trials and overviews. See the Tobacco Addiction Group website for details of the search strategies for these databases.

By searching CENTRAL and the Cochrane Tobacco Addiction Group's register, we will be able to identify any ongoing studies registered in the World Health Organization's portal (www.who.int/ trialsearch) or the US clinical trials register (www.clinicaltrials.gov), and studies reported in Annual Meeting abstracts for the Society for Research on Nicotine and Tobacco (SRNT). We will list in the 'Characteristics of ongoing studies' table any trials which may be candidates for inclusion (i.e. RCTs of smoking cessation interventions using mindfulness approaches with a minimum follow-up of six months), but for which results are not yet available.

\section{Searching other resources}

We will check reference lists of eligible published papers to identify any other relevant papers that may not have been identified by our search, and will consult experts in the field to identify any relevant forthcoming or unpublished research. We will contact the authors of ongoing studies where necessary.

Alongside these manual search strategies, we will employ an automated search strategy developed as part of the HBCP (Michie 2017), using Microsoft Academic. The HBCP aims to improve upon the human ability to synthesise, interpret and deliver evidence on behaviour change interventions, using Natural Language Processing and Machine Learning technologies to automate the extraction, synthesis, and interpretation of findings from behaviour change intervention evaluation reports. We will add any additional studies identified through this method to those found via the manual search, so that we include all relevant evidence. We will perform an evaluation that compares these manual and automated methods of study identification in a subsequent analysis, and will report it in a separate paper. 


\section{Data collection and analysis}

\section{Selection of studies}

Two authors will independently check the titles and abstracts of retrieved studies for relevance, and will acquire full trial reports of those which may be candidates for inclusion. The authors will resolve any disagreements by mutual consent, or by recourse to a third author. Two authors will then independently assess the full texts for eligibility, resolving any disagreements through discussion and with involvement of a third author when necessary. We will classify as 'exclude' any studies for which we obtain full reports, but which do not meet the inclusion criteria. We will record the selection process in sufficient detail to complete a PRISMA flow diagram (Moher 2009) and a 'Characteristics of excluded studies' table, giving reasons for the decision to exclude each study.

\section{Data extraction and management}

Two authors will independently extract study data and compare their findings. We will resolve any disagreements through discussion, involving a third author where necessary. Where available, we will record the following information in the 'Characteristics of included studies' table.

- Methods: study design, study name (if applicable), study dates, country, number of study centres, study setting, study recruitment procedure.

- Participants: N (intervention/control), definition of smoker used, specific demographic characteristics (e.g. mean age, age range, gender, ethnicity, socioeconomic position), mean cigarettes per day, mean Fagerstrom Test for Nicotine Dependence (FTND), relevant inclusion and exclusion criteria.

- Interventions: description of intervention(s) (details of behavioural support and any pharmacological treatment provided), description of control (details of behavioural support and any pharmacological treatment provided), what comparisons will be constructed between which groups.

- Outcomes: relevant primary and secondary outcomes measured, time points reported, biochemical validation, definitions of abstinence, mental health measures used, proportion of participants with follow-up data.

- Details and results of any within-study analyses of moderators of interest: population type; baseline motivation to quit; baseline mental health.

- Notes: funding for trial, and conflicts of interest statements of trial authors (extracted verbatim).

Alongside this data extraction of entities that are typically captured in smoking cessation Cochrane Reviews, we will also perform data extraction using entities of the Behaviour Change Intervention Ontology ( $\mathrm{BCIO}$ ), which is being developed as part of the HBCP (Michie 2017). The ontology consists of granular entities to specify all aspects of behaviour change interventions, such as:

- an intervention's context (including 'Setting' and 'Population');

- content (including 'Behaviour change techniques'; Michie 2013); and

- delivery (including 'Mode of delivery': how an intervention is provided to participants, 'Source': who delivers interventions and 'Schedule': how often an intervention is delivered; Michie 2017).
We will perform an evaluation to compare these methods of data extraction in a subsequent, related paper.

\section{Assessment of risk of bias in included studies}

Two review authors will independently assess the risk of bias for each included study. The version of the 'Risk of bias' (RoB) tool we use will depend on software availability at the time of data extraction. Regardless of what tool we use, we will follow the guidance as set out in the relevant version of the Cochrane Handbook to evaluate the appropriate domains (Higgins 2011 for RoB 1; Higgins 2019 for RoB 2).

For RoB 1, we will assess the following domains (Higgins 2011):

- sequence generation;

- allocation concealment,

- blinding of outcome assessment;

- incomplete outcome data;

- selective reporting; and

- other sources of bias.

However, if RoB 2 is deemed feasible, we will assess studies within the following domains (Higgins 2019):

- bias arising from the randomisation process;

- bias due to deviations from intended interventions;

- bias due to missing outcome data;

- bias in measurement of the outcome; and

- bias in selection of the reported result.

As we are investigating a primarily behavioural intervention, we will not assess the blinding of participants and providers, as it is impossible to blind people to behavioural interventions. This is in accordance with specific guidance from the Cochrane Tobacco Addiction Group.

Each author will record information in trial reports relevant to each domain and then assign each domain as either at low, high, or unclear risk of bias (if using RoB 1) or low, high, or some concerns (if using RoB 2). We will resolve disagreements by discussion with a third review author. We will consider studies to be at high overall risk of bias where we have judged at least one domain to be at high risk; at low overall risk of bias where all domains are judged to be at low risk; and at unclear overall risk of bias (RoB 1) or with some concerns regarding risk of bias ( $R \circ B 2$ ) in all other cases.

\section{Measures of treatment effect}

We will compare quit rates between intervention and comparator groups for each study. We will calculate quit rates on an intentionto-treat basis, including all participants originally randomised to a trial arm. We will treat participants lost to follow-up as relapsed. We will calculate a risk ratio (RR) and 95\% confidence interval $(\mathrm{Cl})$ for each study. We will calculate the RR for each study as: (number of participants that report smoking abstinence in the intervention group/number of participants randomised to the intervention group)/(number of participants that report smoking abstinence in the control (comparison) group/number of participants randomised to the control (comparison) group).

We will also compare any relevant measures of mental health or well-being between intervention and comparator groups for 
each study. We expect most measures of these outcomes to be continuous, in which case we will calculate mean differences (MD) and $95 \% \mathrm{Cls}$ to compare intervention and comparator groups. If studies use different scales for the same outcome (e.g. depression) we will use standardised mean differences (SMD). If any studies measure these outcomes dichotomously, we will summarise the effect size as RRs and $95 \% \mathrm{Cls}$.

\section{Unit of analysis issues}

In the case of cluster-randomised controlled trials, we will extract, where available, a direct estimate of the required effect from an analysis that properly accounts for the cluster design. Where such data are unavailable, we will perform an approximately correct analysis if we can extract the intra-class correlation coefficient (Higgins 2019).

In the case of trials with multiple intervention arms, we will analyse individual arms separately, and split the control arm where necessary to avoid double-counting participants.

\section{Dealing with missing data}

For smoking abstinence, we will assume participants lost to followup to be smoking, as is standard in the field (West 2005). However, we will conduct a sensitivity analysis, excluding numbers lost to follow-up from the denominator. We will calculate mental health and well-being outcomes as complete case analyses, taking into account the potential limitations of this approach.

\section{Assessment of heterogeneity}

In order to assess whether it is appropriate to pool studies and conduct meta-analyses, we will assess the characteristics of included studies to identify any clinical or methodological variance between studies. If we deem the studies to be homogenous enough to be combined meaningfully and we can conduct any meta-analyses, we will assess statistical heterogeneity using the $1^{2}$ statistic. We will consider an $1^{2}$ over $50 \%$ to indicate moderate to substantial heterogeneity. If the $\mathrm{I}^{2}$ is $80 \%$ or more, we will not report a pooled estimate because it could be misleading. We will conduct the subgroup and sensitivity analyses described below to investigate any potential causes of observed heterogeneity.

\section{Assessment of reporting biases}

If we identify and meta-analyse abstinence rates from at least 10 studies for a comparison, we will assess reporting bias using funnel plots. Funnel plots illustrate the relationship between the effect estimates from individual studies against their size or precision. The greater the degree of asymmetry, the greater the potential risk of reporting bias. We will only test for funnel plot asymmetry if there are at least 10 studies in the meta-analysis, as the power would otherwise be too low to distinguish chance from real asymmetry (Higgins 2019).

\section{Data synthesis}

We will provide a narrative summary of the included studies and, where appropriate, conduct meta-analyses.

The primary outcome of abstinence provides dichotomous data, therefore, as per standard methods of the Cochrane Tobacco Addiction Group, we will combine RRs from individual studies using random-effects, Mantel-Haenszel methods, to calculate pooled overall RRs with $95 \% \mathrm{Cls}$.

The primary outcome of mental health and well-being will typically provide continuous data. We will summarise these as the MD or SMD (to allow the combination of different scales measuring the same construct), with $95 \% \mathrm{Cls}$, using inverse variance, randomeffects models. If there is insufficient data to carry out metaanalyses, we will tabulate the existing information and summarise narratively.

We will also narratively report the results of any within-study analyses that have investigated the following moderators:

- population type;

- baseline motivation to quit;

- baseline mental health.

\section{Subgroup analysis and investigation of heterogeneity}

Where it is possible to carry out meta-analyses and we identify sufficient studies, we will investigate potential heterogeneity between studies. We will do this by using subgroup analyses that categorise studies by (broad) intervention type, the type/ intensity of control treatment received, population type, baseline motivation to quit, baseline mental health, number of intervention behaviour change techniques, mode of intervention delivery, and type of therapist. We will compare pooled summary statistics across groups and run statistical tests for subgroup differences.

\section{Sensitivity analysis}

For both primary outcomes, we will test the impact of excluding studies deemed to be at overall high risk of bias. For the abstinence outcome, we will also compare abstinence rates calculated assuming 'missing equals smoking' with abstinence rates calculated through complete case analysis.

\section{Summary of Findings tables and GRADE assessments}

Following standard Cochrane methodology (Higgins 2019), we will create 'Summary of findings' tables including our two primary outcomes (smoking abstinence and mental health and well-being), detailing different comparisons in separate tables. Also following standard Cochrane methodology (Higgins 2019), we will use the five GRADE considerations (risk of bias, inconsistency, imprecision, indirectness and publication bias) to assess the certainty of the body of evidence for each outcome, within each comparison, and to draw conclusions about the certainty of evidence within the text of the review.

\section{ACKNOWLEDGEMENTS}

This protocol was co-authored by employees and editors of the Cochrane Tobacco Addiction Group, which receives infrastructure funding from the National Institute for Health Research (NIHR). The views and opinions expressed therein are those of the authors and do not necessarily reflect those of the Systematic Reviews Programme, NIHR, National Health Service (NHS) or the Department of Health.

We gratefully acknowledge peer review by Margaret A. Maglione, RAND Corporation and $\mathrm{Dr}$ Ana Howarth, Honorary Research Fellow, St George's, University of London; consumer review by 
Lee Bromhead; and editorial review by Dr Jamie Hartmann-Boyce, University of Oxford 


\section{REFERE N CES}

\section{Additional references}

\section{Baer 2003}

Baer RA. Mindfulness training as a clinical intervention: a conceptual and empirical review. Clinical Psychology: Science and Practice 2003;10(2):125-43. [DOI: 10.1093/clipsy.bpg015]

\section{Bishop 2004}

Bishop SR, Lau M, Shapiro S, Carlson L, Anderson ND, Carmody J, et al. Mindfulness: a proposed operational definition. Clinical Psychology: Science and Practice 2004;11(3):230-41. [DOI: 10.1093/clipsy.bph077]

\section{Brewer 2010}

Brewer JA, Bowen S, Smith JT, Marlatt GA, Potenza MN. Mindfulness-based treatments for co-occurring depression and substance use disorders: what can we learn from the brain? Addiction 2010;105(10):1698-706. [DOI: 10.1111/ j.1360-0443.2009.02890.x]

\section{Brewer 2011}

Brewer JA, Mallik S, Babuscio TA, Nich C, Johnson HE, Deleone $\mathrm{CM}$, et al. Mindfulness training for smoking cessation: results from a randomized controlled trial. Drug and Alcohol Dependence 2011;119(1):72-80. [DOI: 10.1016/ j.drugalcdep.2011.05.027]

\section{Chamberlain 2017}

Chamberlain C, O'Mara-Eves A, Porter J, Coleman T, Perlen SM, Thomas J, et al. Psychosocial interventions for supporting women to stop smoking in pregnancy. Cochrane Database of Systematic Reviews 2017, Issue 2. Art. No: CD001055. [DOI: 10.1002/14651858.CD001055.pub5]

\section{Correa-Fernández 2012}

Correa-Fernández V, Ji L, Castro Y, Heppner WL, Vidrine JI, Costello TJ, et al. Mediators of the association of major depressive syndrome and anxiety syndrome with postpartum smoking relapse. Journal of Consulting and Clinical Psychology 2012;80(4):636-48. [DOI: 10.1037/a0027532]

\section{Doll 2004}

Doll R, Peto R, Boreham J, Sutherland I. Mortality in relation to smoking: 50 years' observations on male British doctors. BMJ 2004;328(7455):1519-27. [DOI: 10.1136/bmj.38142.554479.AE]

\section{Glassman 1990}

Glassman AH, Helzer JE, Covey LS, Cottler LB, Stetner F, Tipp JE, et al. Smoking, smoking cessation, and major depression. Journal of the American Medical Association 1990;264(12):1546-9. [DOI: 10.1001/ jama.1990.03450120058029]

\section{Goyal 2014}

Goyal M, Singh S, Sibinga EM, Gould NF, Rowland-Seymour A Sharma R, et al. Meditation programs for psychological stress and well-being: a systematic review and meta-analysis. JAMA Internal Medicine 2014;174(3):357-68. [DOI: 10.1001/ jamainternmed.2013.13018]

\section{Hayes 2016}

Hayes SC, Strosahl KD, Wilson KG. Acceptance and Commitment Therapy. 2nd edition. New York: Guilford Press, 2016.

\section{Heppner 2015}

Heppner WL, Spears CA, Vidrine JI, Wetter DW. Mindfulness and emotion regulation. In: Ostafin BD, Robinson MD, Meier BP, editors(s). Handbook of Mindfulness and Self-Regulation. New York: Springer, 2015:107-20. [DOI: 10.1007/978-1-4939-2263-5_9]

\section{Higgins 2011}

Higgins JP, Green S, editor(s). Cochrane Handbook for Systematic Reviews of Interventions Version 5.1.0 (updated March 2011). The Cochrane Collaboration, 2011. Available from handbook.cochrane.org.

\section{Higgins 2019}

Higgins JP, Thomas J, Chandler J, Cumpston M, Li T, Page MJ, et al, editor(s). Cochrane Handbook for Systematic Reviews of Interventions Version 6.0 (updated July 2019). Cochrane, 2019. Available from www.training.cochrane.org/handbook.

\section{Hölzel 2011}

Hölzel BK, Lazar SW, Gard T, Schuman-Olivier Z, Vago DR, Ott, U. How does mindfulness meditation work? Proposing mechanisms of action from a conceptual and neural perspective. Perspectives on Psychological Science: a Journal of the Association for Psychological Science 2011;6(6):537-59. [DOI: 10.1177/1745691611419671]

\section{Kabat-Zinn 2013}

Kabat-Zinn J. Full Catastrophe Living, revised edition: How to Cope With Stress, Pain and Illness Using Mindfulness Meditation. London: Hachette UK, 2013.

\section{Keng 2011}

Keng S-L, Smoski MJ, Robins CJ. Effects of mindfulness on psychological health: a review of empirical studies. Clinical Psychology Review 2011;31(6):1041-56. [DOI: 10.1016/ j.cpr.2011.04.006]

\section{Linehan 2018}

Linehan MM. Cognitive-behavioral Treatment of Borderline Personality Disorder. New York: Guilford Publications, 2018.

\section{Maglione 2017}

Maglione MA, Ruelaz Maher A, Ewing B, Colaiaco B, Newberry S, Kandrack R, et al. Efficacy of mindfulness meditation for smoking cessation: a systematic review and metaanalysis. Addictive Behaviors 2017;69:27-34. [DOI: 10.1016/ j.addbeh.2017.01.022]

\section{Marchand 2013}

Marchand WR. Mindfulness meditation practices as adjunctive treatments for psychiatric disorders. The Psychiatric Clinics of North America 2013;36(1):141-52. [DOI: 10.1016/ j.psc.2013.01.002] 


\section{Marlatt 2005}

Marlatt G, Donovan DM (editors). Relapse Prevention: Maintenance Strategies in the Treatment of Addictive Behaviors. New York: Guildford Press, 2005.

\section{Michie 2013}

Michie S, Richardson M, Johnston M, Abraham C, Francis J, Hardeman W, et al. The behavior change technique taxonomy (v1) of 93 hierarchically clustered techniques: building an international consensus for the reporting of behavior change interventions. Annals of Behavioral Medicine 2013;46(1):81-95. [DOI: 10.1007/s12160-013-9486-6]

\section{Michie 2017}

Michie S, Thomas J, Johnston M, Aonghusa PM, ShaweTaylor J, Kelly MP, et al. The Human Behaviour-Change Project: harnessing the power of artificial intelligence and machine learning for evidence synthesis and interpretation. Implementation Science 2017;12(1):121. [DOI: org/10.1186/ s13012-017-0641-5]

\section{Moher 2009}

Moher D, Liberati A, Tetzlaff J, Altman DG, the Prisma Group. Preferred reporting items for systematic reviews and meta-analyses: the PRISMA statement. PLoS Medicine 2009;6(7):e1000097. [DOI: https://doi.org/10.1371/ journal.pmed.1000097]

\section{Nhất Hanh 1987}

Nhất Hạnh T. The Miracle of Mindfulness: a Manual on Meditation. Boston: Beacon Press, 1987.

\section{Segal 2002}

Segal ZV, Williams JM, Teasdale JD. Mindfulness-based Cognitive Therapy for Depression: A New Approach to Preventing Relapse. New York: Guilford Press, 2002.

\section{Shiffman 2004}

Shiffman S, West R, Gilbert D, SRNT Work Group on the Assessment of Craving and Withdrawal in Clinical Trials. Recommendation for the assessment of tobacco craving and withdrawal in smoking cessation trials. Nicotine \& Tobacco Research 2004;6(4):559-614. [DOI: 10.1080/14622200410001734067]

\section{Shiffman 2005}

Shiffman S. Dynamic influences on smoking relapse process. Journal of Personality 2005;73(6):1715-48. [DOI: 10.1111/ j.0022-3506.2005.00364.x]

\section{Taylor 2014}

Taylor G, McNeill A, Girling A, Farley A, Lindson-Hawley N, Aveyard $P$. Change in mental health after smoking cessation: systematic review and meta-analysis. BMJ 2014;348:g1151. [DOI: 10.1136/bmj.g1151]

\section{West 2005}

West R, Hajek P, Stead L, Stapleton J. Outcome criteria in smoking cessation trials: proposal for a common standard. Addiction 2005;100(3):299-303. [DOI: 10.1111/ j.1360-0443.2004.00995.x]

\section{West 2017}

West R. Tobacco smoking: health impact, prevalence, correlates and interventions. Psychology \& Health 2017;32(8):1018-36. [DOI: 10.1080/08870446.2017.1325890]

\section{WHO 2019}

World Health Organization. Tobacco. www.who.int/news-room/ fact-sheets/detail/tobacco (accessed 20 May 2020).

\section{APPEN DICES}

\section{Appendix 1. MEDLINE search strategy} MEDLINE-OVID 1946 To present

1. exp Smoking Cessation/

2. exp Tobacco Use Disorder/

3. exp Tobacco Use Cessation/

4. (SMOKING* or TOBACCO or TOBACCO-USE-DISORDER ${ }^{\star}$ or TOBACCO-USE-CESSATION ${ }^{\star}$ or NICOTINE*):MH

5. (SMOKING CESSATION or ANTISMOK $\left.{ }^{\star}\right): T I, A B$

6. (quit* or smok $^{\star}$ or nonsmok ${ }^{\star}$ or cigar $^{\star}$ or tobacco ${ }^{\star}$ or nicotine $\left.{ }^{\star}\right): \mathrm{TI}$

7. smoking cessation.mp.

8. or/1-7

9. exp mindfulness/

10. exp meditation/

11. exp Mind-Body Therapies/ 
12. exp Mind-Body Relations, Metaphysical/

13. exp relation therapy/

14. exp Breathing Exercises/

15. (meditat ${ }^{\star}$ OR mindful ${ }^{\star}$ OR relaxation* mind-body OR body-mind).mp.

16. (Samadhi OR Samapatti):mp.

17. (acceptance adj2 commitment).ti,ab.

18. $\mathrm{Or} / 9-17$

19. 8 AND 18

\section{H I S T ORY}

Protocol first published: Issue 7, 2020

\section{CONTRIBUTIONS OF AUTHORS}

SJ wrote the protocol with input from all authors.

\section{DECLARATIONS OF INTEREST}

SJ: none reported

JB has undertaken research and consultancy for manufacturers of smoking cessation medications (Pfizer and Johnson \& Johnson).

RW has received payments for lectures, research and consultancy from companies that manufacture smoking cessation medications (Pfizer, GlaxoSmithKline and Johnson \& Johnson). He is an unpaid advisor on the Smoke Free smartphone application and to the National Centre for Smoking Cessation and Training.

EN: none reported

JLB: none reported

NL: none reported

All authors declare no financial links with tobacco companies or e-cigarette manufacturers or their representatives.

\section{SOURCES OF SUPPORT}

\section{Internal sources}

- Nuffield Department of Primary Care Health Sciences, University of Oxford, UK Editorial base for the Cochrane Tobacco Addiction Group

\section{External sources}

- Cancer Research UK, UK

SJ's salary was paid by a grant funded by Cancer Research UK (C1417/A22962)

- University College London, UK

JB and RW's salaries were paid by University College London

- National Institute for Health Research, UK

Infrastructure funding for the Cochrane Tobacco Addiction Group 